\title{
Guest Editorial 1
}

\section{INDIA IS LEAPFROGGING TO A NEW ERA IN HEALTHCARE DELIVERY}

Providing healthcare services to 1.33 billion population is indeed a herculean task. India, with its compounding population, coupled with growing geriatric population, has been grappling with the rising triple disease burden, inadequate infrastructure and a low public health, for many years. Today, with increased commitment from the government along with clinical and technological advancements in the sector, India is at the cusp of entering a new era of reformed and re-defined healthcare delivery system. However, to fully gain from these reforms, we need to take certain steps to make the system sustainable while being affordable and accessible.

India has made notable improvements across the spectrum of health and development in the past couple of decades. The average life expectancy has risen steadily from 64 to 68 years between 2005 and 2016. We have reduced our infant mortalities and increased institutional births along with improved immunization coverage throughout the country.

In economic terms, healthcare has become one of India's largest sectors - both in revenue and employment. With burgeoning investments in the sector, it is expected to reach USD 372 billion by 2022 growing at a CAGR of $16 \%{ }^{1}$ The country has also become one of the leading destinations for high-end treatment services along with tremendous capital investment for advanced diagnostic facilities. The hospital and diagnostic centers attracted foreign direct investment (FDI) worth USD 5.25 billion between April 2000 and June 2018. ${ }^{2}$

However, with a score of 41.2 in the Healthcare Access and Quality (HAQ) Index, India still scores way below the global average of 54.4 points. India also lags behind China (48), Sri Lanka (71), Bangladesh (133) and Bhutan (134). ${ }^{3}$

India has one of the lowest public healthcare expenditures in the world, which has just marginally increased from $1.2 \%$ of the GDP in $2013-14$ to $1.4 \%$ in $2017-18 .{ }^{4}$ With an out-of-pocket health expenditure at $62 \%,{ }^{5}$ approximately 63 million poor are pushed into poverty in India every year due to catastrophic health expenditures.

Further, for $16 \%$ of the world's population, we have a disproportionately high share of global disease burden at approx $21 \%$, which is coupled with one of the fastest growing incidences of noncommunicable diseases like hypertension, diabetes, cardiovascular diseases, stroke, and cancer as well as mental illness and trauma.

The World Bank estimates that $90 \%$ of all health needs can be met at the primary healthcare level. However, primary health care in India is grossly underinvested in the country. For secondary and tertiary care, hospital bed density per 10,000 people at 7 is way below the global average of $21.6{ }^{6}$ In addition to the financial and physical resource constraint, another major concern is the shortage of skilled health workforce at all levels across the continuum of care.

Nevertheless, we cannot undermine the fact that given India's population and disease burden, developing the required infrastructure and provisioning for health care for all needs a massive economic as well as political commitment.

Although the Government of India, over the years, has launched various nationwide programs for prevention, control and eradication of communicable and noncommunicable diseases and improvement of maternal and child health; the launch of 'Ayushman Bharat' program is set to be a game-changer for the healthcare sector in India. The program with its two pillars - health and wellness centers (HWCs) and Pradhan Mantri Jan Arogya Yojna (PMJAY), world's largest public funded health insurance scheme - will help India enter into a completely new era of reformed and re-defined healthcare delivery system.

Successful implementation of 'Ayushman Bharat' will not only facilitate in achieving India's Sustainable Development Goal (SDG) - three targets, but also enhance health outcomes, spur investments in health sector as well as generate new job opportunities.

However, to build a truly healthy nation, India needs re-engineering of its healthcare delivery system at systemic, financial as well as infrastructural levels. It is imperative that a new paradigm is evolved in healthcare policy, program and practice that aim to rationalize costs while expanding access and reducing the need for advanced care. At the fundamental level, the focus needs to shift from 'sick' care to 'health' care through preventive, promotive and accountable care.

Our healthcare ecosystem needs to be more patient-focused by demonstrating greater sensitivity towards patients, understanding their expectations and engaging with them. We need to embed patient-centricity in designing and execution of core operating processes and systems to enable patients to get appropriate quality care. 
The private healthcare sector, that has been providing nearly $60 \%$ of in-patient care and has contributed to $70 \%$ of bed capacity expansion in the last decade, will be a crucial partner in attaining this. The government can utilize the private sector expertise in skill development and infrastructure expansion as well as its CSR funds towards these endeavors. Appropriate and efficient collaboration between the government and the private sector will form the bedrock for effective implementation of all programs and strategies.

Considering the current healthcare demands and massive coverage expected under the Ayushman Bharat, we would need to substantially expand the network of healthcare facilities and add approximately 0.64 million beds with an investment of INR 3 trillion (USD 44 billion) over next 10 years. With limited public funds, government needs to provide appropriate long-term financing options along with incentives and tax benefits to the private sector to be able to contribute effectively.

While the government is working towards incentivizing accreditation and implementing standard treatment guidelines to attain quality of care, it is also crucial to formulate viable and sustainable strategies for healthcare cost reimbursement to the private sector. The government needs to develop a framework for standard package definition as well as reimbursement tariff determination that is based on a scientific costing methodology, with the ability to evolve in response to changing contexts, revised priorities and experiences gained over the years. Time-driven Activity-based Costing (TDABC) is a proven method for determining the actual costs incurred within the healthcare delivery system to arrive at rational reimbursement tariffs for healthcare services. ${ }^{7}$ The time has come to revisit the low tariff rates of procedures and diagnostics that were based on old CGHS rates. Also, the government should embrace quality by having differential tariff rates based on set parameters of quality that include the qualification and experience of the healthcare personnel and the quality of the equipment used in different hospitals. This is very much akin to the differential pricing mechanism prevailing in government-provided services like Indian Railways. It must be remembered that the land prices and cost of capital are higher in urban areas but the availability of qualified doctors, nurses and paramedical staff is a great challenge in semi-urban and rural surroundings that are also plagued with lack of infrastructure that includes availability of potable drinking water and 24 hours electricity.

India also needs to invest substantially in its healthcare workforce. While there is a need to increase the number of healthcare professionals, enhancing quality and skill development of existing workforce is also critical. We need focused and appropriate skilling, re-skilling as well as up-skilling programs for existing as well as additional manpower - doctors, nurses and allied healthcare professionals.

With acute shortage of specialist doctors in the country, we need to take some immediate steps like increasing PG seats, both in district hospitals and private healthcare facilities, creating layered Certificate and Bridge Courses and developing a credit accumulation framework. Further, the conversion of 58 District Hospitals to medical colleges needs to be fast-tracked and focus on setting up of medical colleges in rural areas with appropriate incentives for doctors and faculty needs to be prioritized.

Healthcare technology has been playing a crucial role in ensuring early diagnostics and improving precision of treatment and efficiency in care delivery. In future also, technology-enabled interventions such as remote medical advice, health call-centers, tele-radiology, artificial intelligence, emergency response and e-learning platforms, will play a significant role in ensuring timely access to quality health care. Government's plans should include and implement digitization and innovative health IT solutions even at the lowest level to ensure easy verification and monitoring of delivery and avoidance of fraud in health care at the ground level.

While working towards implementation of Electronic Health Record (EHR) Standards in the country, the government must also encourage adoption of information technology for all clinical establishments. New innovative IT solutions and app-based systems that are user-friendly should be adopted in a way that even small clinical establishments can also use them.

Trauma care systems in India are at a nascent stage of development and there exists a disparity between trauma care services available in different parts of the country - from rural health posts where staff do not have training as doctors to tertiary care centers staffed by specialists. We need national level strategies to reduce the burden of road traffic injuries and mortalities by improving and strengthening the existing system of trauma care in India, with system interventions for prehospital, hospital and posthospital care. Surgeons, anesthesiologists and provision of good blood banking services within $50 \mathrm{~km}$ of an accident shall be the pillars of these services.

Further, there is a need to increase the effectiveness of continuum of care in the country by strengthening linkages between primary, secondary and tertiary institutions. While we build and strengthen the primary healthcare system, we need to define and standardize our referral system across the continuum of care as well as between public and 
private providers. This will not only help in enhancing the health outcomes, but also aid in cost-effective utilization of resources at each level.

In the past few years, public-private partnership (PPP) has been the preferred approach for collaborations between the government and private players. However, various challenges have been faced in execution of PPP projects. While the government is working towards strengthening and formalizing the PPP framework for the country, they need to create a conducive environment to make PPP more attractive and effective. There should be clear objectives and deliverables for the private partners and they should get greater autonomy to function within those targets.

The Central Government has been pushing the states to adopt the Clinical Establishment Act (CEA) which will help regulate the healthcare sector, which is crucial in order to achieve quality across the country. However, the implementation panel of CEA needs to include representatives from doctor's community and the private sector to create a balanced approach in its implementation and avoid a situation like License Raj.

Lastly, we need to encourage and recognize transparency, self-regulation and third-party ratings and going forward rewarding outcomes to help bridge the widening trust-deficit in the sector. Recently, the Federation of Indian Chambers of Commerce and Industry (FICCI) with the help of NATHEALTH has developed the Code of Ethics for the Health Services Industry, through extensive consultation with stakeholders. The Code encourages members to voluntarily and collectively commit to ethical professional conduct for patient care and is applicable to healthcare professionals, healthcare providers, diagnostic centers and other healthcare institutions operating in India. Government should mandate all the hospitals joining PMJAY to implement this Code.

\section{Conclusion}

India has already taken the road to help redefine and restructure delivery of healthcare services in the country. The above steps will further help to strengthen our capacities for providing affordable, accessible and sustainable care to the entire population. The country needs effective health system governance through a mix of regulation and encouragement through incentives for the private sector along with patient-centricity as the underlying principle.

Brig Dr Arvind Lal Chairman and Managing Director, Dr Lal PathLabs Chair, FICCI Health Services Committee

\section{REFERENCES}

1. Source: India Brand Equity Foundation

2. Source: DIPP, GoI

3. https://www.business-standard.com/article/current-affairs/india-worse-than-bhutan-bangladesh-in-healthcare-ranks145th-globally-118052400135_1.html

4. https://economictimes.indiatimes.com/industry/healthcare/biotech/healthcare/indias-spending-on-health-sector-hasgrown-nadda/articleshow /65309487.cms?from $=$ mdr

5. Source: Data published by SEARO, WHO

6. http://www.searo.who.int/india/mediacentre/events/world_health_day/universal_health_4april2018.pdf

7. FICCI Study titled 'Demystifying Healthcare Costs: A Scientific Approach', 2018 